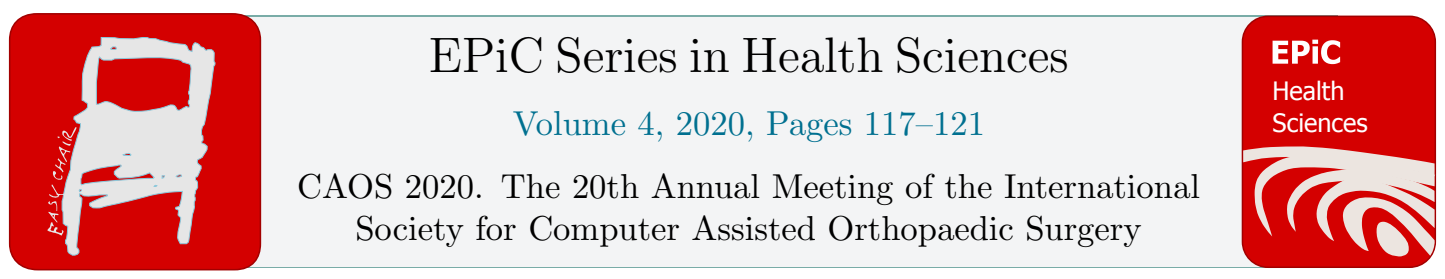

\title{
Augmented Active Shape Model Search - towards 3D Ultrasound-based Bone Surface Reconstruction
}

\author{
Benjamin Hohlmann and Klaus Radermacher \\ Chair of Medical Engineering, Helmholtz-Institute f. BME, RWTH Aachen University of \\ Technology, Aachen, Germany \\ hohlmann@hia.rwth-aachen.de, radermacher@hia.rwth-aachen.de
}

\begin{abstract}
Patient-specific instrumentation in total knee arthroplasty (TKA), among other medical indications, requires a three-dimensional model of the bones involved. Currently, these are typically segmented from computer tomography images. Ultrasound offers a cheap as well as radiation-less imaging alternative, but suffers from a low signal-to-noise ratio as well as several other image artifacts. The interleaved partial active shape models search (IPASM) adapts a general physiological model to a set of images of a single patient, but suffers from false correspondences being soft tissue interfaces that are interpreted as bone surface. In order to counter this problem, a convolutional neural network $(\mathrm{CNN})$ is applied to preprocess ultrasound images into bone confidence maps. This reduces the average surface distance error in an in-vivo evaluation by 0.7 to $1.3 \mathrm{~mm}$.
\end{abstract}

\section{Introduction}

Some diagnostic approaches as well as patient-specific biomechanical modelling or patient specific instruments and implants respectively require a three-dimensional model of the patient's knee joint [16]. The gold standard for 3D imaging of bone is computed tomography (CT), which however is associated with additional radiation and high costs. Alternative approaches proposed for 3D reconstructions of bone are MRI image based [7] or based on bi-planar x-ray imaging with model based reconstructions $[8,9]$. These approaches are associated with high costs, too, or show limited accuracy in specific clinical applications. In contrast, ultrasound images are radiation-less, comparably cheap and commonly available for chair-side imaging in orthopedic clinical routine. However, the extraction of the bone surfaces is more complex. The knee joint consists of multiple bones, partially occluding each other. Thus it is not possible to acquire the surface of all portions of the knee joint with just one volumetric ultrasound image. Instead, multiple images need to be registered while some parts of the bone surface remain invisible and requires its augmentation with model based a-priori knowledge. In 
order to reconstruct a 3D model from an ultrasound datasets, the task of segmentation is therefore complemented by registration and interpolation.

Statistical Shape Models (SSM) combined with the active shape model (ASM) search offer the ability to register a model to an image, solving the problem of registration and interpolation. These techniques are common practice in various fields and provided promising results [10,11]. An extended version of the ASM, the interleaved partial active shape model search (IPASM) allows for registration in a multi-view setting [12]. While this approach works reasonably well for in-vitro experiments [13], a detailed in-vivo analysis revealed a high number of false correspondences: Soft tissue interfaces appear very similar to bone surfaces in ultrasound images and are falsely matched with the model. This problem could be solved by an explicit segmentation of the bone surface, prior to the registration and model adaptation.

Recent publications on medical image segmentation focused on the application of convolutional neural networks $(\mathrm{CNN})$ in various architectures. Published accuracy of a combined phase symmetry and $\mathrm{CNN}$ approach for ultrasound segmentation of the distal femur reaches $0.2 \mathrm{~mm}$ of average surface distance error [14]. Combinations of SSMs with CNNs were successfully applied to similar problems. Ambellan et al. proposed an image processing pipeline for the automatic reconstruction of 3D knee joint models from magnetic resonance images (MRI), including the bones as well as the cartilage [15].

Consequently, the aim of this study was to improve the reconstruction accuracy by augmenting the IPASM pipeline with an additional CNN preprocessing.

\section{Methods}

The backbone of our application is the IPASM algorithm [12]. First, a statistical shape model (SSM) is built from several hundred healthy knees. This model is then adapted to the patient's anatomy, which is captured by several volumetric ultrasound images. The algorithm determines an update of the models surface according to the surrounding voxel values and alternates between rigid registration and shape adaption to accomplish the surface update.

For preprocessing of the ultrasound images into bone confidence maps, we use the Pyramid Attention Network (PAN), a state-of-the-art semantic segmentation network. See [16] for implementation details. The segmentation is multiplied with the original US image to obtain a bone confidence map. The architecture was trained on 3323 training images of four distal femora recorded and annotated by our group. Due to the high variance of the bone surface in arbitrarily oriented 2D BMode images of the 3D bone shape, we expect the network to generalize well to unseen bone shapes. We opted for a segmentation of the bone surface only, in order to prevent false classification of pitch black areas due to other occlusions. See Figure 1 for an example. The images have a fixed size of 381 $\mathrm{x} 465$ pixels and a pixel spacing of $0.1 \mathrm{~mm}$.

For reconstruction of a knee joint, roughly registered volumetric images of the patient are required. Our setup includes a mechanically swiping 3D US probe (4DL14-5/38, Ultrasonix, Peabody, USA). The image processing as well as the synchronization of tracking and ultrasound hardware is performed by a small application, running on a standard PC. After acquisition, the volumes are sliced and the bone confidence maps are inferred by our CNN.

The in-vivo experiment includes 12 volumetric images of one proband's right knee that were manually registered to the ground truth, segmented from an MRI. The ground truth segmentation as well as registration was performed with 3D Slicer, an open source tool [17]. The low number of images induces very challenging conditions, where large parts of the distal and dorsal bone surface are not visible. As the proband's knee is very similar to the mean shape, we further simulate patients with bigger or smaller femora by altering the initial model: Instead of starting from the mean shape, we 
initialize the first three modes of the SSM with \pm two standard deviations, varying the size as well as the aspect ratio of the model.

Three settings are evaluated: First, the initial fit of the statistical shape model to the patient's bone. Second, the fit after application of the IPASM algorithm on the original ultrasound images and third, the fit after preprocessing with a CNN and application of the IPASM.

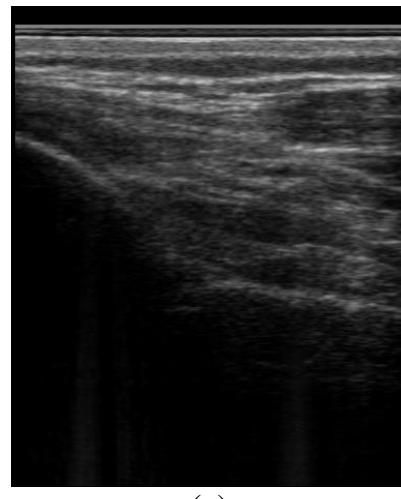

(a)

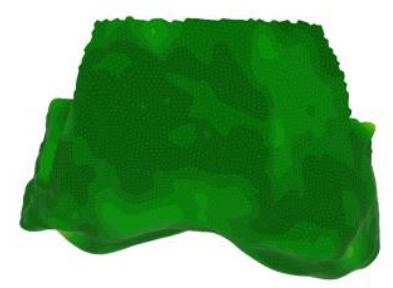

(d)

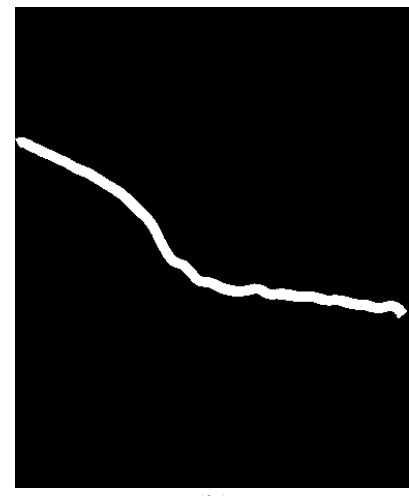

(b)

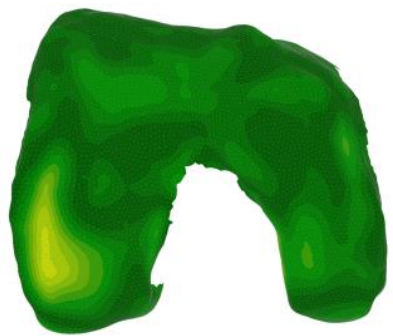

(e)

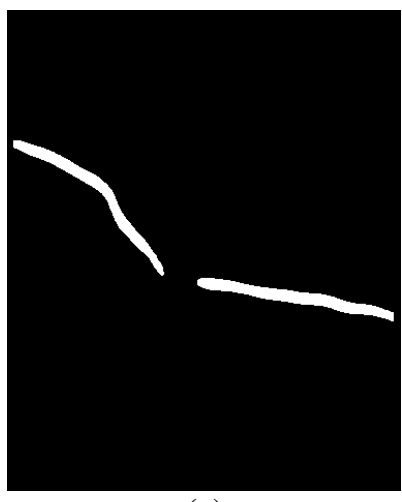

(c)

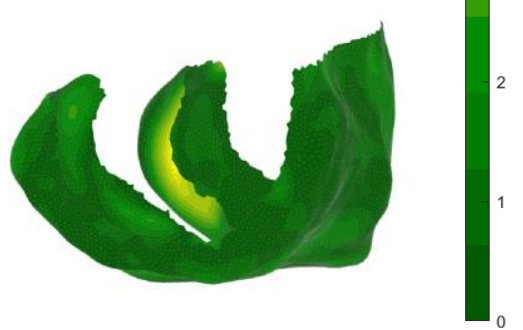

(f)

Figure 1: Top row: An ultrasound volume slice of our validation set (a), its ground truth annotation (b) and the predicted bone mask of our network (c). Bottom row: Heatmap visualization of the surface distance error (SDE) of the 'enlarged' experiment. Frontal (d), distal (e) and lateral (f) view. Notice the two spots with high reconstruction error occur in areas not visible in the ultrasound images.

\section{Results}

Training of the CNN took 12 hours on an NVidia GTX 1070 GPU. The segmentation error on the validation dataset reached its minimum after 86 epochs. Results of the full reconstruction pipeline can be found in Table 1. The first row shows the initial average as well as maximal surface distance error. The second and third row show the error after application of the IPASM and CNN + IPASM, respectively. Comparing the mean shape to the ground truth, the error decreases slightly. For the shrank, as well as the enlarged shape, the reconstruction benefits noticeably from the CNN segmentation. In all cases, the average SDE remains below $1.3 \mathrm{~mm}$. 


\begin{tabular}{lcccccr}
\hline SDE $(\mathrm{mm})$ & \multicolumn{2}{c}{ Original Shape } & \multicolumn{2}{c}{ Shrank Shape } & \multicolumn{2}{c}{ Enlarge Shape } \\
& Avg. & Max. & Avg. & Max. & Avg. & Max. \\
\hline Initial Mean Shape & 0.72 & 3.57 & 2.38 & 10.7 & 2.68 & 8.75 \\
IPASM & 0.87 & 4.29 & 1.58 & 7.39 & 1.92 & 8.24 \\
CNN + IPASM & 0.84 & 4.04 & 1.27 & 4.87 & 1.09 & 7.07 \\
\hline
\end{tabular}

Table 1: Average and maximal surface distance error (SDE) of the reconstructed model.

\section{Discussion}

The CNN preprocessing improved the reconstruction in all cases. As it had no impact for the original sized model, we hypothesize that the errors encountered here are not due to segmentation issues. Instead, a lower limit for the reconstruction error of occluded surfaces is reached. For a simulated small and big knee, the average as well as maximal error could be reduced significantly. Wrong soft-tissue correspondences found when using the original ultrasound images can be corrected. As can be seen for the 'enlarged' experiment in Figure 1, the remaining maximal errors of $4.87 \mathrm{~mm}$ and $7.07 \mathrm{~mm}$ occur in occluded areas of the distal as well as posterior condyles and can be attributed to wrong extrapolation.

Compared to a 2D-3D reconstruction of the distal femur on the basis of SSMs and bi-planar X-rays [18], the root mean square error of our approach is slightly lower, $1.61 \mathrm{~mm}$ in the worst case compared to $1.68 \mathrm{~mm}$, without exposing the patient to radiation. Other publications on SSM based reconstruction from ultrasound images of the femur report a much higher error of $3.5 \mathrm{~mm} \mathrm{[19].}$

As shown in previous studies [13], the registration is highly relevant for the overall reconstruction. Future work will therefore investigate an additional refinement of the registration using the segmented images, as well as freehand tracked 2D probes for bigger image volumes.

\section{References}

1. Smoger LM, Fitzpatrick CK, Clary CW et al. (2015) Statistical modeling to characterize relationships between knee anatomy and kinematics. J Orthop Res 33(11): 1620-1630. doi: 10.1002/jor.22948

2. Asseln M, Hanisch C, Al Hares G et al. (2016) Automatic parameterisation of the distal femur based on 3D surface data: a novel approach for systematic morphological analysis und optimisation. Bone \&amp. Joint Journal Orthopaedic Proceedings Supplement

3. Asseln M, Al Hares G, Eschweiler J et al. (2014) Development of a patient-specific musculoskeletal model of the knee for clinical application and kinematic validation based on invivo measurements. In: Orthopaedic Proceedings, vol 96, p 20

4. Fitzpatrick CK, Clary CW, Rullkoetter PJ (2012) The role of patient, surgical, and implant design variation in total knee replacement performance. Journal of biomechanics 45(12): 20922102

5. Asseln M (2019) Morphological and Functional Analysis of the Knee Joint for Implant Design Optimization, 1. Auflage. Aachener Beiträge zur Medizintechnik, vol 57. Shaker, Düren

6. Changhai Ding, Patrick Garnero, Flavia Cicuttini et al. (2005) Knee cartilage defects: association with early radiographic osteoarthritis, decreased cartilage volume, increased joint surface area and type II collagen breakdown. Osteoarthritis and Cartilage 13(3): 198-205. doi: 10.1016/j.joca.2004.11.007 
7. Al Hares G, Eschweiler J, Radermacher K (2015) Combined magnetic resonance imaging approach for the assessment of in vivo knee joint kinematics under full weight-bearing conditions. Proc Inst Mech Eng H 229(6): 439-451. doi: 10.1177/0954411915585863

8. Fleute M, Lavallée S (1999) Nonrigid 3-D/2-D Registration of Images Using Statistical Models. In: Taylor C, Colchester A (eds) Medical Image Computing and Computer-Assisted Intervention - MICCAI'99. Springer Berlin Heidelberg, Berlin, Heidelberg, pp 138-147

9. M. R. Mahfouz, W. A. Hoff, R. D. Komistek et al. (2003) A robust method for registration of three-dimensional knee implant models to two-dimensional fluoroscopy images. IEEE Trans Med Imaging 22(12): 1561-1574. doi: 10.1109/TMI.2003.820027

10. Anas EMA, Seitel A, Rasoulian A et al. (2016) Registration of a statistical model to intraoperative ultrasound for scaphoid screw fixation. International journal of computer assisted radiology and surgery 11(6): 957-965

11. Bock T de, Tadross R, Mahfouz M et al. (2012) Automatic Real-Time Reconstruction of Patient-Specific 3D Knee Model Using Ultrasound RF Data. In: Orthopaedic Proceedings, vol 94, p 40

12. Hänisch C, Hohlmann B, Radermacher K (2017) The interleaved partial active shape model search (IPASM) algorithm - Preliminary results of a novel approach towards 3D ultrasoundbased bone surface reconstruction. In: Radermacher K, Rodriguez Y Baena F (eds) CAOS2017. EPiC Series in Health Sciences, vol 1, Aachen, Germany, pp 399-406

13. Hohlmann B, Radermacher K (2019) The interleaved partial active shape model -IPASMsearch algorithm - towards 3D ultrasound-based bone surface reconstruction. In: Patrick Meere, Ferdinando Rodriguez Y Baena (eds) CAOS 2019. The 19th Annual Meeting of the International Society for Computer Assisted Orthopaedic Surgery, vol 3. EasyChair

14. Alsinan AZ, Patel VM, Hacihaliloglu I (2019) Automatic segmentation of bone surfaces from ultrasound using a filter-layer-guided CNN. International journal of computer assisted radiology and surgery 14(5): 775-783. doi: 10.1007/s11548-019-01934-0

15. Ambellan F, Tack A, Ehlke M et al. (2019) Automated segmentation of knee bone and cartilage combining statistical shape knowledge and convolutional neural networks: Data from the Osteoarthritis Initiative. Medical image analysis 52: 109-118. doi: 10.1016/j.media.2018.11.009

16. Li H, Xiong P, An J et al. (2018) Pyramid Attention Network for Semantic Segmentation. https://arxiv.org/pdf/1805.10180

17. Jólesz FA (ed) (2014) Intraoperative imaging and image-guided therapy. Springer, New York, NY

18. N. Baka, B.L. Kaptein, M. de Bruijne et al. (2011) 2D-3D shape reconstruction of the distal femur from stereo X-ray imaging using statistical shape models. Medical image analysis 15(6): 840-850. doi: 10.1016/j.media.2011.04.001

19. Dean C. Barratt, Carolyn S.K. Chan, Philip J. Edwards et al. (2008) Instantiation and registration of statistical shape models of the femur and pelvis using 3D ultrasound imaging. Medical image analysis 12(3): 358-374. doi: 10.1016/j.media.2007.12.006 